\title{
Analysis of the Current Situation and Trends of Online Education Research Based on VOSviewer
}

\author{
Siyu Liu \\ The Attached Middle School to Jiangxi Normal University, Nanchang, China \\ Email: Liusiyu20050102@icloud.com
}

How to cite this paper: Liu, S. Y. (2021) Analysis of the Current Situation and Trends of Online Education Research Based on VOSviewer. Open Journal of Social Sciences, 9, 426-432.

https://doi.org/10.4236/jss.2021.99030

Received: August 26, 2021

Accepted: September 14, 2021

Published: September 17, 2021

Copyright $\odot 2021$ by author(s) and Scientific Research Publishing Inc. This work is licensed under the Creative Commons Attribution-NonCommercial International License (CC BY-NC 4.0). http://creativecommons.org/licenses/by-nc/4.0/

\begin{abstract}
With the widespread use of the Internet, online education is getting more and more attention. This paper collects online education literature collect in Web of Science from 2018 to 2020 and analyzes it in three aspects: co-author network, co-occurrence network, and co-citation network, showing the current status of online learning in China in recent years. Based on network education can fully display teaching information and resources, form special learning websites and virtual communities, and provide a collaborative learning environment for teachers and students. Through the monitoring and management of the subnetwork, students' learning experience and status can be recorded. It is meaningful for understanding the research hot topics of online education in China and equally valuable for understanding the collaboration patterns and citation status of Chinese scholars in this field.
\end{abstract}

\section{Keywords}

Online Education, Constructivist Learning Theory, Problem Analysis

\section{Introduction}

Online education began in the mid-1990s. With the development of society and the advancement of science and technology, online education has become a very novel form of education. In the last two years, the global epidemic has led to the development of online learning. Many schools and colleges have been unable to teach offline in this scenario and have made a hasty transition to online learning, and many online education platforms have entered in the public vision (Chen Zibo et al., 2014). Since the educational system developed based on WAP by online educational institutions to now exploring the needs of lifelong learners, the "Tower of Experience" theory published by Dell in the audio ovisual teaching stage in 1946 laid a good foundation for educational technology-media tool theory. 
With the development of online education, a large number of scholars have conducted research on online education and published a large number of papers in important academic journals. These studies reveal the current situation of the development of online education in China and the problems brought about and to be faced by the development of online education. Based on the high-quality papers, this paper uses the VOS VIEWER tool to analyze the development of online education in China. In order to have a clear understanding of the current status of online education research in China.

\section{Data Sources and Analysis Tools}

\subsection{Data Sources}

The core collection of the Web of Science database was selected as the data source, and 19,390 articles were retrieved under the topic path of "online education" with the time span of 2018-2020. From the search result, we selected "PEOPLES R CHINA" from "Country/Region" in the refined search results on the left side, and then obtained 3802 papers on online education in China. These 3802 papers are the data used in this study

\subsection{Analysis Tools}

Vosviewer is a software tool for creating maps based on network data and for visualizing and exploring these maps. The functionality of Vosviewer can be summarized as follows: 1) maps based on network data. 2) Although Vosviewer is intended primarily for analyzing bibliometric networks, it can be used to create, visualize, and explore maps based on any type of network data. From 2014-2015, Liu Xianjun and his team conducted a survey on Chinese college education, and put forward two important development directions of academic and application. Vosviewer has become an important tool for knowledge mapping research on scientific data in recent years.

\section{Data Analysis}

Select Vosviewer as the tool to analyze the data, and first select "create a map based on bibliographic data" in creating to create a visual network of co-author (Cheng Qikai et al., 2019), co-occurrence, and co-citation relationships. Then, select "read data from bibliographic database files" in Select data source. After selecting, import the data downloaded from Web of Science.

\subsection{Co-Author Analysis}

A collaboration network is a hot research area in complex network analysis, which is a network formed by the cooperation relationship between scientists and research institutions. In this network, researchers or research institutions are abstracted as a node and their collaborative relationships (joint publications, etc.) are abstracted as lines, thus forming a complex network of research collaboration. As the complexity of scientific problems increases, collaborative research 
among global scholars has become a way to produce high-quality research results. Looking at the collaborative network of authors in a field reveals the depth, intensity, cliques, and major contributors of collaboration in that field.

To observe the collaborative network of high publication authors, only authors with at least 5 papers in Web of Science were selected for this study, which can be set in Vosviewer. To do this, we first imported the 3802 papers collected above, and then in Vosviewer, in the "choose type of analysis and counting method" screen, we selected "type of analysis", select "co-authorship", "unit of analysis", "authors ", select "full counting" in "counting method", and then select "minimum" in "choose threshold". In "minimum number of documents of an author", select 5, and in "minimum number of citations of an author", select 0 . Then in the "choose number of cited authors" screen, select not to show all 260 items that are not linked to other authors. Finally, in the "verify selected authors" screen, select all options and click finish. The author collaboration network is obtained (see Figure 1).

\subsection{Co-Occurrence Analysis}

The graph is composed of 19 groups of subnetworks formed by 188 authors posting together, generating a total of 261 links, with a total strength of 414 links between them. The most representative of them is the network formed by the core of Wang Wei, Zhou Pan and Liu Yang. Wang Wei's 27 articles and other

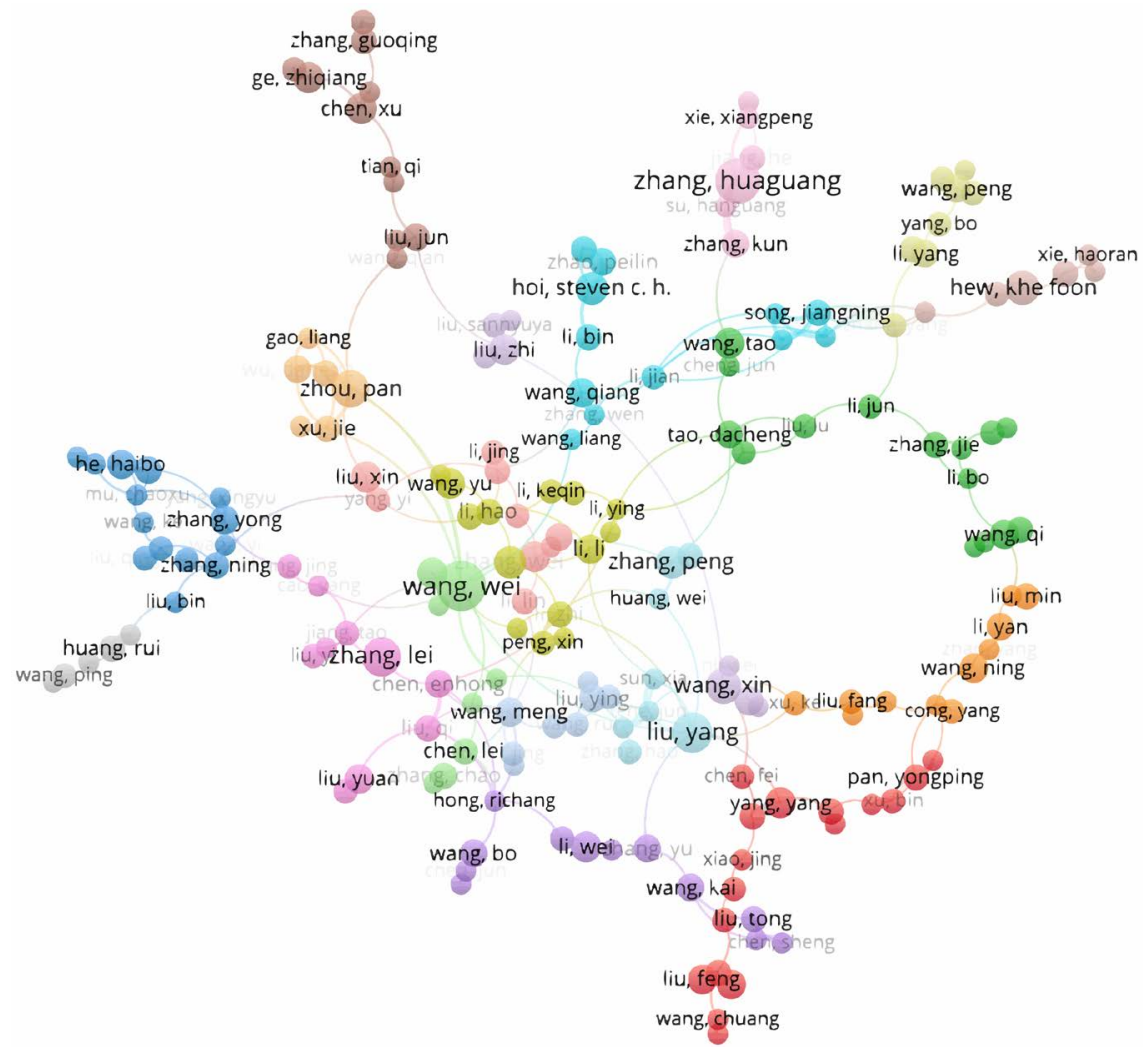

Figure 1. Online education author partnership network. 
authors form 11 subnetworks, generating 13 links; Wang Wei is an important node, and all other nodes are connected to him, with him as the center, showing a center-outward diffusion pattern. 15 articles of Zhou Pan and other authors form 16 subnetworks, generating 7 links; 17 articles of Liu Yang and other authors formed 15 groups of subnetworks and 9 links. The links derived from them also have a significant proportion in the figure.

It is clear from Figure 1 that the authors of articles on online education in China with a higher number of articles generate a similarly high number of subnetworks and connections (Guan \& Li, 2014). At the same time, this figure clearly shows that collaboration among key investigators is more prevalent. However, simple small networks of a few individuals account for the majority of the collaborative networks of principal investigators, and large complex collaborative networks have not yet been formed.

In literature citation, keywords are defined as words and terms selected from the literature that encompass and represent the topics, methods, and information items of the full text. If a pair of keywords appear together in the same document, they are considered to have a co-occurrence relationship, and the cooccurrence strength is equal to the number of documents containing these keywords. The greater the co-occurrence intensity, the stronger the connotation correlation between two words and the better the consistency on the topic, and the associated network calculation of keyword co-occurrence can reflect the structure and evolution law of the research topic, for this reason, this paper constructs the co-word relationship network with online education data.

In Vosviewer, select "type of analysis" in the "choose type of analysis and counting method" screen, select "co-occurrence", "all keywords" in "unit of analysis", and "counting method". In "counting method", select "full counting". After that, select 5 for "minimum number of occurrences of a keyword" in "choose threshold". Finally, in the "verify selected keywords" screen, select all options and click finish to ignore all items that are not connected to other authors in the 260 items. All items that are not linked to other authors are ignored. graph of the co-word network is obtained. (See Figure 2)

Figure 2 consists of a total of 925 words and eight subgroups. The total number of links is 23,515 , and the total strength of their connections is 35,671 . The most representative words are "model" "deep learning" "algorithm" "classification" "performance" "design" "optimization" "machine learning". There is also a strong connection between these most frequently repeated words.

The word "model", for example, has only 2 subnets, but forms 296 links with a total strength of 1255, making it the most frequently mentioned word among all. At the same time, "model" is also closely related to other words that are mentioned frequently. Such as, "deep learning", "algorithm”, "classification”, etc. are mentioned above. At the mean time, "performance" is not only related to "model", but also to "students" "technology" and "design". This is not just a showing of how every word is inextricably linked to each other, but it is also clear that 


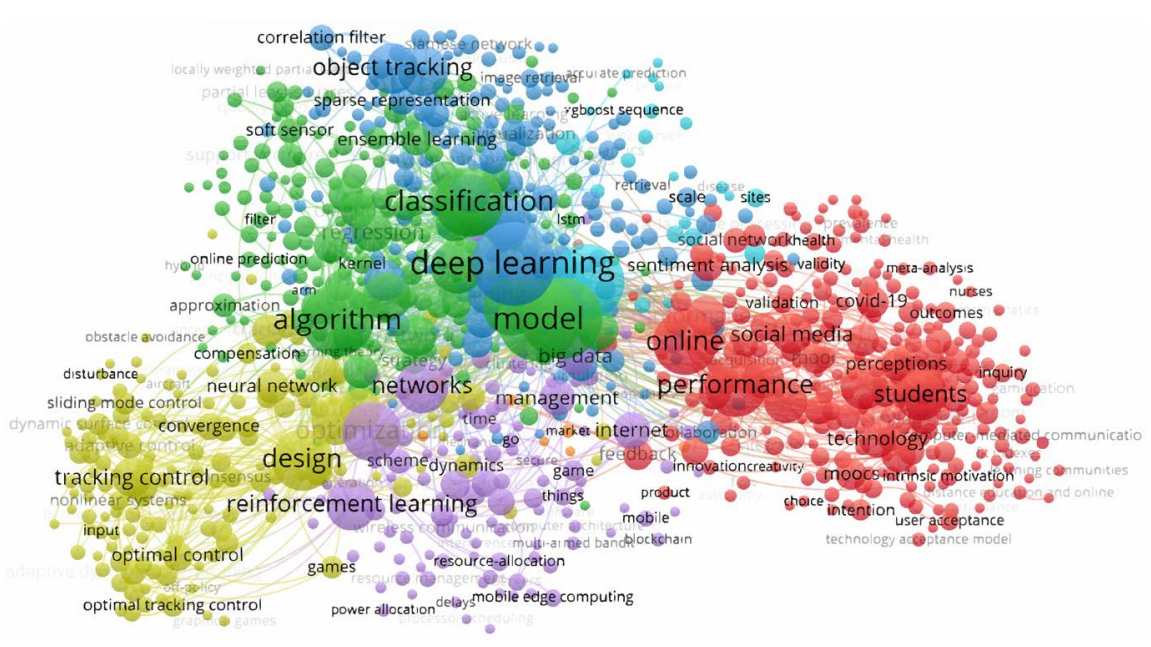

Figure 2. Online education and co-occurrence network.

words that are mentioned more frequently in this chart are more likely to be more closely related to other words. Unlike the co-authorship network above, the co-word network is more tightly connected and complex (Zhu Yonghai et al., 2014), suggesting that scholars are more unified in their choice of research topics. In recent years, research has focused more on machine learning, algorithms, and other technologies. Co-citation network analysis can reveal relationship between literature and thus enable automatic clustering of literature relevance.

\subsection{Co-Citation Analysis}

Citation analysis has been used as a method to reveal the intrinsic characteristics and patterns of scientific journals and papers, to evaluate the academic influence of scientific journals and papers, and predict the trends in the development of related disciplines. Citation literature refers to literature that cites a paper, reflecting the continuation, application, development, or evaluation of the research work of that paper; co-cited literature refers to literature that is cited as a reference at the same time as a paper, reflecting it as a common basis for further research with that paper (Chen Zibo et al., 2014).

In Vosviewer, after completing the steps in Section 3.2. In the "Choose the type of analysis and counting method" screen, select "co-citation", "cited references" in "unit of analysis", and "counting method", select "full counting". Afterwards, select 20 in the "Choose threshold" field and 213 in the "Choose number of cited references" field. Finally, select 213 in the "Verify selected cited references" field. In the "verify selected cited references" screen, select all the options and click Finish to obtain the citation network (see Figure 3).

The co-citation network is composed of 4 subnetworks of 213 items, forming a total of 7404 links, and the total strength of their connections is 42,453 . Figure 3 differs from Figure 1 and Figure 2 in that it is a horizontal graph, in which the group centered on "fornell c, 1981, j marketing res, v18, p39, doi". The group 


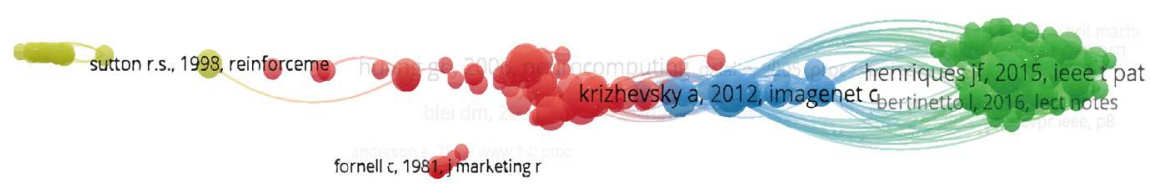

Figure 3. Online education and co-citation.

centered on "10.2307/3151312" has no connection with the other groups, but only with the articles on the right side of the figure, forming a small closed network, which shows that most of the authors' citations are concentrated on a very small number of articles. In contrast, the articles on the left side of the figure have only a few simple links or no links. Among the integrated co-citation networks, "krizhevsky a, 2012, imagenet classificat, v25, p1097, doi 10. 1145/ 2065386" is the document with the highest link strength, with 156 links formed between it and the other items. The total strength was 1450, and this article was cited 161 times. This article is also from the right side of this graph.

\section{Conclusion}

From the analysis of three aspects: author collaboration network, co-word network, and co-citation network, it is found that the total number of online education publications in China is relatively small. Among 3802 papers, only 448 scholars published more than 5 online education papers, accounting for less than $12 \%$ of the total. Author collaboration is not yet sufficient, with 260 of the 448 not collaborating with others and the other 188 forming a collaborative network dominated by a spindle-shaped two-person collaboration. The research themes in Chinese education, however, have greater relevance (Wang Ying et al., 2014), with research interests focusing on technologies such as machine learning and algorithms, which is a completely different change from the focus of past research in online education. In addition, in the analysis of co-cited literature, the citation behavior of Chinese scholars is not seen to be more scattered, and cocitations are only concentrated on individual foreign literature.

Although Chinese online education research is relatively weak in terms of the number of papers, the intensity of collaboration, and high quality citations, the research themes have changed significantly, with new technologies such as artificial intelligence being emphasized and focused on, which will benefit the development of online education in China. With the emphasis on teaching and learning in Chinese higher education and the influence of the epidemic factor at the same time, online education will draw more and more attention, and more scholars will focus on online learning in the future.

\section{Conflicts of Interest}

The author declares no conflicts of interest regarding the publication of this paper.

\section{References}

Chen, Z. B., Zuo, J., \& Wang, M. Q. (2014). A Brief Discussion on the Current Situation 
of Online Education Industry Development and Product Design Research. Contemporary Education Practice and Teaching Research, No. 12, 66.

Cheng, Q. K., Wang, J. M., \& Lu, W. (2019). A Study of Domain-Based Vocabulary Discovery Based on Citation Co-Word Networks. Data Analysis and Knowledge Discovery, No. 6, 57-65.

Guan, J., \& Li, Q. T. (2014). The Current Situation, Trends and Experiences of Online Education Development in China. Chinese E-learning, No. 8, 62-66.

Zhu, Y. H., Han, X. B., Yang, J., \& Cheng, J. G. (2014). The Irreversible Trend of Online Development in Higher Education: A Comprehensive Analysis and Insights from the 11-Year Series of Reports on Online Education in the United States. Tsinghua University Education Research, 35, 92-100.

Wang, Y., Liu, J. D., \& Han, Y. H. (2014). Theoretical Thinking and Practical Exploration on the Development of MOOCs in China. China's Electro-Chemical Education, No. 1, 52-60. 